\title{
Genre et attitudes de stigmatisation à l'égard des Personnes vivant avec le VIH (PVIH) au Bénin
}

\author{
Affo Mingnimon Alphonse ${ }^{1}$, Djogbenou Yao Robert ${ }^{1,2}$, Acotcheou Pacôme ${ }^{1}$ \\ \& Tossou Cocou Rigobert ${ }^{1}$ \\ ${ }^{1}$ Centre de Formation et de Recherche en matière de Population (CEFORP) de l'Université \\ d'Abomey-Calavi (UAC), Bénin \\ ${ }^{2}$ Département de Démographie, Université de Montréal, Canada \\ Pour les correspondances : amaffo@gmail.com
}

\section{Résumé}

Contexte : Les attitudes de rejet des $\mathrm{PVVIH}$ constituent un frein à l'accès universel et à leurs droits essentiels. Cet article vise à apporter un éclairage sur les disparités de genre en matière de stigmatisation des PVVIH.

Méthodologie : Analyse secondaire des données de l'EDSB 201 I-2012. Un indicateur de niveau de stigmatisation a été créé pour faire des analyses multi-variées de régression logistique sur un échantillon de 4813 hommes et I 4849 femmes.

Résultats : Pour les deux sexes, avoir un niveau élevé d'instruction, être exposé fréquemment aux médias, être chrétien et appartenir au groupe ethnique Adja déterminent l'attitude bienveillante envers les PVVIH. II en est de même pour les femmes qui sont en union conjugale, celles qui vivent en milieu rural, celles vivant dans des ménages riches ou celles n'ayant aucune activité économique.

Conclusion: Développer des stratégies de protection des PVVIH en s'appuyant sur les attitudes bienveillantes observées.

Mots clés : stigmatisation, genre, VIH/Sida, Bénin

\section{Abstract}

Background: The rejectionist attitudes of PLHIV are a barrier to universal access and their basic rights. This article aims to shed light on gender disparities in the stigmatization of PLHIV.

Methodology: Secondary analysis of the 20I I-20I2 EDSB data. A stigma level indicator was created to perform multi-varied logistic regression analyzes on a sample of 4813 men and I 4849 women.

Results: For both sexes, having a high level of education, being exposed frequently to the media, being a Christian and belonging to the Adja ethnic group determine the caring attitude towards PLHIV. It is the same for women who are in a conjugal relationship, those who live in rural areas, those who live in wealthy households or those who have no economic activity.

Conclusions: Develop protective strategies for PLHIV based on the caring attitudes observed.

Keywords: stigma, gender, HIV / AIDS, Benin

\section{Introduction}

Les travaux récents sur le $\mathrm{VIH} / \mathrm{Sida}$ révèlent une amélioration sensible en matière de prévention et de traitement dans le monde même si l'Afrique de l'Ouest et du Centre continue d'enregistrer des difficultés dues aux comportements des personnes à hauts risques qui étaient déjà mises à l'écart du fait de leur statut avant leur exposition au risque du $\mathrm{VIH}$. D'après un rapport de Médecin sans frontière (2016), bien qu'ayant une prévalence moyenne considérée comme relativement faible au $\mathrm{VIH}(2,3 \%)$, la région de l'Afrique de l'Ouest et du Centre abrite 6,6 millions de $\mathrm{PVVIH}$ représentant près du cinquième
$(\mathrm{I7,9 \% )}$ du fardeau mondial du $\mathrm{VIH}$ en $20 \mathrm{I} 4$ avec $21 \%$ de nouvelles infections et $45 \%$ de nouvelles infections chez les enfants. Ces résultats traduisent une contribution de la région de $36 \%$ au décès dans le monde alors que la tendance mondiale des décès liés au Sida est en déclin.

$\mathrm{Au}$ Bénin, les résultats de l'enquête démographique et de santé réalisée en 20II-20I2 (INSAE et ICF International, 20I3) révèlent une prévalence globale du $\mathrm{VIH}$ de I,2\% avec plusieurs disparités. On note une prévalence plus élevée chez les femmes $(\mathrm{I}, 4 \%)$ comparées aux hommes $(\mathrm{I}, 0 \%)$. 
II en est de même en milieu urbain ( $1,6 \%)$ comparé au milieu rural $(0,9 \%)$. Le même rapport indique qu'une plus forte proportion de femmes $(36,7 \%)$ a fait le test du $\mathrm{VIH}$ comparées aux hommes (13,2\%). Les données de surveillance de l'infection à $\mathrm{VIH}$ au Bénin indiquent par ailleurs une prévalence de I,6\% chez les femmes enceintes avec une disparité selon le milieu de résidence (urbain : $1,8 \%$; rural : 0,8\%). L'ensemble de ces données renforce le caractère féminin de l'infection au Bénin (Ministère de la Santé, 2018).

En affectant l'individu, le $\mathrm{VIH} / \mathrm{Sida}$ touche également sa famille ce qui atteste que les besoins des PVVIH ne se limitent pas seulement à une prise en charge biomédicale ; les soutiens psychologique, social et spirituel peuvent également les aider à réduire la stigmatisation à laquelle elles sont confrontées et qui font qu'elles se sentent exclues de la société (Zongo et al., 2009). Le mépris ou l'intolérance constitue donc l'un des obstacles à la lutte contre le $\mathrm{VIH} /$ Sida notamment l'accès universel aux programmes de prévention et de traitement. L'histoire de la maladie (Sida) renseigne qu'une place prépondérante était accordée aux aspects biologiques au détriment des questions éthiques et sociales particulièrement celles qui relèvent du respect des droits des Personnes Vivant avec le VIH $(\mathrm{PVVIH})$. En plus du fardeau que constitue le $\mathrm{VIH} /$ Sida, les PVVIH sont exposées au rejet, ce qui accentue leur culpabilisation, parfois celle de leur entourage familial (RAP+ Afrique de l'Ouest et du Centre et ONUSIDA, 2002 ; INSAE et ICF International, 2013, op.cit.). Comme dans la plupart des pays d'Afrique de l'Ouest, les problèmes liés à la sexualité au Bénin sont le plus souvent entourés de tabous fondés sur des représentations et discours parfois dévalorisants, ce qui complexifie davantage leur résolution. L'un des exemples les plus connus est celui des infections sexuellement transmissibles (y compris le $\mathrm{VIH} /$ Sida) dont l'apparition chez un individu s'accompagne souvent de risque d'ostracisme. Une telle situation amène les $\mathrm{PVVIH}$ à s'isoler et à faire parfois de fausse déclaration sur leur statut sérologique créant ainsi un frein supplémentaire pour leur prise en charge adéquate et exposant du coup leurs partenaires à des risques de contagion encore plus élevés. Les conséquences de l'intolérance à l'égard des $\mathrm{PWVIH}$ sont d'abord d'ordre psychosocial avant d'être socioéconomique, épidémiologique, etc. Elles se traduisent par des affects psychologiques, une dégradation de l'environnement socioaffectif et une préoccupation pour la vie du couple et la procréation (RNP+, 20I2 ; Beaulieu, 20|4).

D'après l'ONUSIDA, la stigmatisation et la discrimination ont largement été des obstacles au test 4751 de dépistage du $\mathrm{VIH}$, la prise en charge correcte des $\mathrm{PWIH}$ et la prévention de la maladie. Elles sont manifestes dans tous les pays et régions du monde par toutes sortes de facteurs, notamment une mauvaise compréhension de la maladie, les fausses perceptions sur la transmission du $\mathrm{VIH}$ ainsi que la mauvaise information sur la réalité du Sida, etc. (ONUSIDA, 2005). Face aux comportements de rejet des PVIH au Bénin, les pouvoirs publics ont pris des mesures législatives et réglementaires qui visent à faire respecter les Droits de ces personnes. Ainsi, naquit la Loi №2005-3I du 10 Avril 2006 portant prévention, prise en charge et contrôle du $\mathrm{VIH} /$ Sida en République du Bénin (Bénin, 2006). Cette disposition renforce l'article 36 de la Loi $\mathrm{N}^{\circ} 90$ 32 du II décembre 1990 portant Constitution de la République du Bénin qui prescrit le respect et la considération de toute personne sans discrimination aucune en vue du maintien de la paix et de la cohésion nationale (Bénin, 1990). Par ailleurs, les $\mathrm{PWIH}$ sont encouragées à se constituer en association pour la défense de leurs droits et à promouvoir leur vie socioprofessionnelle avec l'appui des pouvoirs publics et des organisations de la société civile. A terme, le Bénin vise comme le souhaite I'ONUSIDA un accès universel aux activités préventives et curatives du $\mathrm{VIH}$ pour réduire sensiblement les cas de nouvelles infections, de décès liés au Sida ainsi que les actes de stigmatisation à l'égard des PVIH. Dans ce sens, il a été mis en œuvre des interventions pour l'accès universel (prévention, soins et traitements et soutien) qui couvrent plusieurs engagements de I'ONUSIDA. Cependant, les défis qui s'imposent relèvent en partie de l'insuffisance des ressources humaines, matérielles et financières pour satisfaire les besoins que requièrent les chantiers ouverts dans ce domaine. Par ailleurs, le personnel en poste ne jouit pas forcément d'un statut susceptible de pérenniser leurs interventions au sein de leur unité de travail (CNLS, 2016). Si certains travaux (Nzié, 20I0, Beaulieu, 2014 ; Ky-Zerbo et al., 20I4, Novignon et al., 20I4), ont mis en relief les facteurs explicatifs de la stigmatisation associée au $\mathrm{VIH} / \mathrm{Sida}$, les présentes analyses apportent la lumière sur les disparités de genre en rapport les $\mathrm{PVVIH}$.

\section{Objectif}

L'objectif général poursuivi est de déterminer les facteurs qui favorisent les attitudes de stigmatisation à l'égard des $\mathrm{PVVIH}$ au sein des femmes et des hommes au Bénin. 


\section{Revue de littérature et cadre théorique Revue de littérature}

La stigmatisation associée au $\mathrm{VIH} / \mathrm{Sida}$ s'entend généralement comme tout comportement ou attitude qui vise à jeter de l'opprobre sur un individu présumé porteur d'un vice $(\mathrm{VIH})$ ou tendant à lui faire subir une réprobation sociale parce qu'il a contrevenu à une norme. La discrimination qui en est l'une des manifestations les plus concrètes peut se réaliser par une attitude malveillante ou un comportement de mépris tel une agression verbale, physique, la privation/restriction d'un service ou d'un emploi à la PWVIH alors qu'elle en a droit (Bruyn, 1998 ; Desclaux, 200I). La stigmatisation se manifeste sous deux formes : (i) une forme interne et souvent peu perceptible appelée autostigmatisation qui traduit l'intériorisation de la $\mathrm{PVVIH}$ des réactions négatives des membres de son entourage (les autres) à son égard pour la crétiniser, l'humilier ou l'éviter et (ii) une forme externe imputée exclusivement à l'altérité et qui se manifeste par une attitude ou un comportement négatif d'ostracisme à son égard. Bruyn (1998, op.cit.) a identifié cinq facteurs de stigmatisations associées au $\mathrm{VIH}$ : la mort, la contamination, les relations sexuelles, la consommation de drogues injectables et le rejet des $\mathrm{PWVIH}$. Ce dernier facteur entretenu par certaines croyances religieuses ou morales traduit un moindre engagement communautaire, parfois la désinformation et freine l'application des lois protégeant les PVVIH dans beaucoup de pays. La stigmatisation peut s'étendre parfois au personnel de santé et aux établissements médicaux ce qui oblige certains patients à rechercher des traitements loin de leur cadre habituel de vie. C'est pourquoi le souci de confidentialité sous-tend une implication sélective des soignants dans la prise en charge des $\mathrm{PWVIH}$ (Zongo et Ouattara, 2008 ; Médecin sans frontière, 2016). D'après I'ONUSIDA la stigmatisation et la discrimination restreignent la volonté des personnes à connaître leur statut sérologique et limitent le recours au dépistage. Elles favorisent également le déni de la maladie (Sida) et freinent le recours des $\mathrm{PWVIH}$ au traitement. Le même rapport révèle que plus de la moitié des PVVIH déclarent en être victimes en Chine, au Paraguay et au Rwanda (ONUSIDA, 20I0).

La stigmatisation concerne l'individu mais aussi son entourage et contribue au retard de dépistage à cause de la peur qu'elle suscite (Skinner et Mfecane, 2004). Or, la connaissance précoce du statut sérologique au $\mathrm{VIH}$ joue un rôle majeur dans la prise en charge, la prévention et l'adoption des comportements sexuels sains chez les $\mathrm{PVVIH}$ (Beaulieu, 2014, op.cit.).
Pour réduire les risques de mortalité liés au $\mathrm{VIH} /$ Sida, I'ONUSIDA a mis sur pied depuis 2005 un programme d'intensification de l'accès au traitement anti rétroviral (TAR) avec des résultats qui se sont traduits par une diminution de $35 \%$ des décès liés au Sida au sein de 16 millions de PVIH dans le monde dont les trois quarts sont en Afrique subsaharienne. Le Bénin dispose depuis 200I d'un programme d'accès aux Anti rétroviraux (ARV) dont la phase opérationnelle a démarré en 2002 à Cotonou avant d'être progressivement mise à l'échelle à travers une centaine de sites de prise en charge du VIH. Le programme d'accélération de la riposte au $\mathrm{VIH} / \mathrm{Sida}$ au Bénin a permis de mettre $37 \%$ de PVIH sous traitement en 2015. Au regard des progrès enregistrés dans le cadre du TAR, I'ONUSIDA envisage, l'accélération de la riposte contre le $\mathrm{VIH}$ afin qu'à l'horizon $2020,90 \%$ des $\mathrm{PVVIH}$ connaissent leur statut $\mathrm{VIH}, 90 \%$ des $\mathrm{PVVIH}$ reçoivent un TAR continu et $90 \%$ de toutes les personnes sous traitement atteignent une suppression virale (ONUSIDA, 2015). En attendant de parvenir à cette fin, les disparités sur les regards empreintes de stéréotypes et de craintes portés par l'entourage sur les PVVIH se maintiennent par endroits. II importe de les élucider pour permettre aux décideurs d'apporter des solutions adéquates à ces situations qui ne sont pas moins une violation des Droits humains. Le Bénin est considéré comme un pays à épidémie $\mathrm{VIH}$ mixte car ayant une prévalence faible $(1,2 \%)$ au sein de la population globale mais élevée $(15,0 \%)$ au sein des groupes à haut risque (CNLS, 20I6). Si des mesures ne sont pas prises pour réduire les risques à tous les niveaux, le pays restera toujours vulnérable à une épidémie généralisée.

Au plan empirique, peu de travaux ont été réalisés sur la stigmatisation et la discrimination associée au $\mathrm{VIH} /$ Sida au Bénin. Les travaux qui se sont intéressés à ce sujet en Afrique ont rapporté les facteurs explicatifs des attitudes de tolérance vis-à-vis des $\mathrm{PVVIH}$. En effet, les facteurs socioculturel, socioéconomique, sociodémographique et institutionnel ont été identifiés comme les plus associés au phénomène (Zongo et al., 2009 ; Nzié, 2010 ; KyZerbo et al., 2014 ; Trinitapoli, 2015 ; INSAE et UNICEF, 20I5).

\section{Cadre théorique}

Le cadre d'analyse s'inscrit dans le courant de l'interactionnisme en particulier, les relations entre identités sociales (réelle et virtuelle) qui se jouent entre deux acteurs dans la détermination mutuelle des comportements des PVVIH et des autres personnes de leur environnement (Le Breton, 20I2). D'après Rostaing (2015), le stigmate est l'attribut qui rend l'individu différent de la catégorie dans laquelle 
on voudrait le classer. La stigmatisation s'analyse en terme relationnel et renvoie autant à la catégorie proprement parler qu'aux réactions sociales qu'elle suscite et aux efforts du stigmatisé pour y échapper. II identifie trois types de stigmate : (i) les monstruosités du corps marqués par diverses difformités ; (ii) les tares du caractère qui aux yeux d'autrui, prennent l'aspect d'un manque de volonté, de passions irrépressibles ou antinaturelles, de croyances égarées et rigides, de malhonnêteté, et dont on infère l'existence chez un individu parce que l'on sait qu'il est ou a été déviant et (iii) les stigmates tribaux qui peuvent se transmettre de génération en génération et contaminer également tous les membres d'une famille. D'après Goffman (1975), les stigmatisés peuvent être discrédités ou discréditables selon qu'ils laissent apparaître ou dissimulent leur stigmate. Benoist (2007) retient que la question de la stigmatisation n'est pas neutre. Elle procède d'une forme particulière de conscience, sans laquelle elle demeure invisible. Elle se fonde sur une vision du monde qui traduit l'éthique dont elle procède. II s'agit parfois d'un jeu symétrique où le regard de l'un (supposé venu d'en haut) écrase celui qu'il rencontre (et qui se croît faible). Se sentant du coup transformé par le regard de l'autre, ce dernier éprouve davantage de difficultés dans l'élaboration de son schéma corporel et peut à l'opposer, réagir pour devenir aussi stigmatisant refusant ainsi l'autostigmatisation. Certaines investigations mettent en relief le caractère prépondérant de l'autostigmatisation dans les interactions entre les $\mathrm{PVVIH}$ et leur entourage qualifié de "normaux ». Parmi ces personnes dites "normaux » peuvent figurer d'autres PVVIH non déclarées ou non connues. Nous désignons ici tous ceux qui sont en interaction avec la PVIIH par l'expression " autres ». Ayant réalisé un travail de recherche sur $219 \mathrm{PVVH}$ au Burkina Faso, Ky-Zerbo et al. (2014) ont identifié l'autostigmatisation comme la forme majeure de stigmatisation ( $46 \%$ de $\mathrm{PVVIH} \mathrm{l'ayant} \mathrm{éprouvé} \mathrm{contre}$ $40 \%$ de stigmatisation dans les relations interpersonnelles et II\% dans les services de santé). Par ailleurs, les interactions entre les personnes portant des stigmates et les "autres" sont généralement marquées par un certain malaise partagé par chacune des parties.

Sous-produit de la déviance, la stigmatisation évolue en fonction du contexte social dans lequel elle prend forme. Benoist (2007, op.cit.) a mis en évidence une corrélation forte lorsque la discrimination porte sur des traits physiques ou sur une maladie comme le Sida. II soutient que la stigmatisation s'enracine dans trois champs : (i) la peur ; (ii) l'anomalie et (iii) la régulation sociale. Elle se manifeste à travers des stéréotypes d'inquiétudes, voire de menaces potentielles, qui au lieu d'être pensées en termes de probabilité le sont en termes de certitudes d'une part et faisant de tout empreinte un caractère indélébile dont l'individu ne peut plus en sortir. L'un des enjeux de la stigmatisation est la difficulté à concilier l'identité sociale réelle de la personne stigmatisée et son identité virtuelle marquée par les attentes de la société à son égard. La stigmatisation résulte d'une interaction entre des rôles plutôt qu'entre des individus ce qui amène la personne stigmatisée à reproduire le jeu de l'interaction, l'ensemble des préjugés relevant de l'altérité : fragilité, honte, affliction (Alpe et al., 2005). II ressort de tout ce qui précède que la stigmatisation n'existe pas en soi, elle est le résultat d'interactions entre des attributs personnels et des stéréotypes. Pour mettre en évidence la dimension genre de la stigmatisation des $\mathrm{PVVIH}$, nous postulons l'hypothèse selon laquelle, le niveau de tolérance à l'égard des PVIH est influencé par les facteurs institutionnels, le contexte de résidence, les facteurs culturels, socioéconomiques, démographiques, la connaissance du $\mathrm{VIH} / \mathrm{Sida}$ et l'exposition aux médias.

\section{Données et méthodes \\ Données}

Les données exploitées proviennent de la quatrième édition de l'Enquête Démographique et de Santé du Bénin (EDSB 20ll-20l2) réalisée par l'Institut National de la Statistique et de l'Analyse Economique (INSAE) en collaboration avec Macro International ICF. La population cible est constituée de 19662 répondants (I4849 femmes âgées de I5-49 ans et 4813 hommes de I5-64 ans) ayant entendu parler du $\mathrm{VIH} / \mathrm{Sida}$ et qui ont été interrogés dans le cadre des questions relatives à la stigmatisation des $\mathrm{PVVIH}$.

\section{Définition des variables}

La stigmatisation est définie comme un processus par lequel un jugement négatif est porté sur une personne ou un groupe de personnes en raison des attributs qu'elles possèdent (ou qu'ils sont supposés posséder) et qui les rend différents des autres (RNP+, 20/2). La discrimination qui en est l'une des manifestations se révèle par un traitement inégal ou injuste infligé à une personne ou un groupe de personnes étiquetées comme déviants par rapport aux normes admises. Ce cliché peut concerner les relations sociales de manière générale, le cadre associatif, les structures de soins, etc. (RNP+, 20I2, op.cit.).

Variable dépendante :Il s'agit du niveau de stigmatisation et de discrimination à l'égard des $\mathrm{PWVIH}$. Pour la mesurer, il a été construit un 
indicateur composite : l'Échelle d'Attitudes Stigmatisantes envers les PVVIH (EASE-PVVIH). D'après Beaulieu (20I4, op.cit.), l'EASE-PVVIH constitue au plan psychométrique, un outil fiable et valide pour mesurer les attitudes stigmatisantes associées au VIH. Dans le cadre de l'enquête, l'indicateur a été construit à partir de cinq items (questions) issus des outils de collecte. II a été demandé aux répondants ayant entendu parler du $\mathrm{VIH} /$ Sida quelle attitude ils adopteraient s'ils étaient confrontés aux situations suivantes impliquant les PVVIH : (i) prendre soin chez soi d'un parent ayant contracté le $\mathrm{VIH}$; (ii) acheter des légumes frais chez un commerçant vivant avec le $\mathrm{VIH}$; (iii) autoriser une enseignante ayant contracté le $\mathrm{VIH}$ mais qui n'est pas malade à continuer à travailler ; (iv) garder secret l'état d'un membre de la famille ayant contracté le $\mathrm{VIH}$ et $(\mathrm{v})$ partager la nourriture avec une personne vivant avec le $\mathrm{VIH}$. L'indicateur ainsi conçu comporte trois modalités : (i) aucune tolérance des cinq attitudes ; (ii) accord avec au moins une attitude bienveillante et (iii) accord avec les cinq attitudes bienveillantes.

\section{Variables indépendantes}

Parmi les variables indépendantes considérées à partir de la revue de la littérature figurent celles-ci contre : (i) connaissance du VIH/Sida ; (ii) milieu de résidence ; (iii) niveau de vie du ménage ; (iv) niveau d'instruction ; (v) occupation principale ; (vi) ethnie ; (vii) religion ; (viii) âge ; (xix) exposition aux médias et $(x)$ état matrimonial. Les taux de couverture de chacune de ces variables retenues sont tous à $100 \%$ (cf. tableau $\mathrm{Al}$ en annexe) ce qui atteste de leur bonne qualité pour conduire les analyses.

\section{Méthodes}

Trois niveaux d'analyse ont été effectués.Premièrement, des analyses descriptives univariées ont été utilisées pour décrire la distribution en pourcentage de l'échantillon par variable d'étude. Ensuite, les analyses bivariées (tableaux croisés avec le test de Khi deux) ont été effectuées pour décrire les associations entre le niveau de stigmatisation et de discrimination à l'égard des PVVIH et chacune des variables indépendantes. Enfin, une modélisation logistique multinomiale a été utilisée pour calculer les rapports de risques relatifs (Relative Risk Ratio : RRR) avec des intervalles de confiance de $95 \%$ ), entre chacune des variables indépendantes et le niveau de tolérance envers les $\mathrm{PVVIH}$. Le choix de cette méthode est justifié par l'objectif de l'étude et la nature qualitative à trois modalités de la variable dépendante.
Spécification du modèle multinomial

Cette méthode d'analyse multi-variée réservée aux variables qualitatives de plus de deux modalités repose sur l'idée selon laquelle une variable à expliquer représente les possibilités de choix d'un individu parmi $\mathrm{M}+\mathrm{I}$ possibilités (modalités) et donc ses préférences. La régression logistique multinomiale est donc une généralisation de la régression logistique binomiale sur des variables à expliquer à plusieurs modalités. Contrairement au modèle binomial où un seul vecteur de paramètres $\beta$ suffit pour déterminer les deux probabilités, puisque $\operatorname{Pr}\left(y_{i}=0\right)+\operatorname{Pr}\left(y_{i}=1\right)=1$, dans le modèle multinomial, plusieurs vecteurs de paramètres $\beta_{\mathrm{j}}$ différents pour chaque modalité sont nécessaires pour estimer la probabilité de la réalisation d'une modalité par rapport à la modalité de référence. Les coefficients de ce modèle sont toujours relatifs à la modalité de référence de la variable dépendante. Dans un modèle multinomial à $M+I$ modalités, la probabilité du choix j pour l'individu i est donnée par l'expression :

$$
\begin{aligned}
P\left(y_{i}=j\right)= & \frac{\exp \left(x_{i} a_{j}\right)}{\sum_{k=0}^{M} \exp \left(x_{i} a_{k}\right)} \\
& =\frac{1}{1+\sum_{\substack{k=0 \\
k \neq j}}^{M} \exp \left(x_{i}\left(a_{k}-a_{j}\right)\right)}
\end{aligned}
$$

Avec $x_{i}$ le vecteur des caractéristiques de l'individu $i$ et $a_{j}$ le vecteur caractéristique de la modalité $j$. Dans ce modèle, les probabilités s'écrivent de la façon suivante :

$$
\begin{aligned}
& P\left(y_{i}=0\right) \\
& =\frac{1}{1+\exp \left(x_{i}\left(a_{1}-a_{0}\right)\right)+\exp \left(x_{i}\left(a_{2}-a_{0}\right)\right)} \\
& P\left(y_{i}=1\right) \\
& =\frac{1}{1+\exp \left(x_{i}\left(a_{0}-a_{1}\right)\right)+\exp \left(x_{i}\left(a_{2}-a_{1}\right)\right)} \\
& P\left(y_{i}=2\right) \\
& =\frac{1}{1+\exp \left(x_{i}\left(a_{0}-a_{2}\right)\right)+\exp \left(x_{i}\left(a_{1}-a_{2}\right)\right)}
\end{aligned}
$$

Comme la variable dépendante a trois modalités : (i) aucune tolérance des cinq attitudes ; (ii) accord avec au moins une attitude bienveillante et (iii) accord avec les cinq attitudes bienveillantes, seuls les coefficients $\alpha_{2}, \alpha_{3}$, seront estimés dans le modèle et on fixe $\alpha_{1}$. Ceci implique que les risques observés sont relatifs à la catégorie " aucune tolérance des cinq attitudes ". Nous voulons expliquer ici, l'accord des répondants avec au moins une attitude bienveillante envers les $\mathrm{PWVIH}$ et leur accord avec les cinq attitudes bienveillantes à l'égard des $\mathrm{PVVIH}$ par rapport à " aucune tolérance des cinq attitudes " qui est la modalité de référence. 


\section{Résultats}

\section{Description de l'échantillon}

Le tableau I ci-dessous décrit les différentes caractéristiques de l'échantillon chez les femmes et les hommes. L'échantillon total pour l'analyse comprenait 14849 femmes et 4813 hommes. Globalement, $48 \%$ des femmes et $47 \%$ des hommes résident en milieu urbain contre $52,1 \%$ des femmes et $53,1 \%$ des hommes en milieu rural. En ce qui concerne le niveau de vie du ménage, respectivement $32,2 \%$ et $31,5 \%$ des femmes et des hommes vivent dans les ménages pauvres contre $49 \%$ des femmes et des hommes dans les ménages riches chacun. Environ $60,5 \%$ des femmes et $56,1 \%$ des hommes sont chrétiens contre $20 \%$ des femmes et $22,2 \%$ des hommes musulmans. Par ailleurs, plus de la moitié des femmes $(56,6 \%)$ et plus du tiers $(34,5 \%)$ des hommes sont sans niveau d'instruction contre respectivement $25 \%$ et $41,3 \%$ des femmes et des hommes ayant le niveau secondaire ou plus. Environ $35 \%$ des femmes appartiennent aux catégories d'âges 15-24 ans et 25-34 ans chacune. Par contre, chez les hommes, respectivement $31,3 \%$ et $30 \%$ sont dans les catégories d'âges de 15-24 ans et 35-49 ans. Globalement, la majorité des répondants était exposée aux médias plus d'une fois par semaine (46,9\% des femmes et $63 \%$ des hommes). Enfin, quel que soit le sexe des répondants, plus de $60 \%$ sont en union contre $24 \%$ des femmes et $35 \%$ des hommes célibataires.

Tolérance des hommes et des femmes ayant entendu parler du Sida à l'égard des PVVIH

Comme l'indique le tableau 2, l'attitude bienveillante la plus courante exprimée à l'égard des $\mathrm{PVVIH}$ est le fait « de savoir qu'on peut partager la nourriture avec une PVVIH sans contracter le VIH ". Elle est exprimée par $67,7 \%$ des femmes et $76,9 \%$ des hommes. Par contre, celle la moins partagée est " l'achat des légumes frais auprès d'un marchand ou vendeur PVVIH » exprimée par $40 \%$ des femmes et $44 \%$ des hommes. Considérant le niveau de tolérance, le graphique I révèle que la proportion de répondants qui se montreraient tolérants dans les cinq attitudes bienveillantes réunies citées envers les $\mathrm{PVVIH}$ est deux fois moins élevée chez les femmes (6\%) comparées aux hommes (14\%). Lorsqu'il s'agit de l'une au moins des cinq attitudes bienveillantes, plus de femmes (89\%) que d'hommes $(83 \%)$ se montreraient tolérantes à l'égard des $\mathrm{PVVIH}$.

\section{Variations différentielles du degré de stigmatisation et de discrimination à l'égard des PVIH}

Le tableau 3 décrit l'association entre le degré de stigmatisation et de discrimination et les variables 4755 indépendantes. II révèle qu'aussi bien chez les hommes que chez les femmes, le milieu de résidence, le niveau de vie du ménage, le niveau d'instruction, l'occupation principale, l'exposition aux médias, la religion et l'ethnie sont significativement associées au degré de tolérance envers les $\mathrm{PVVIH}$. II en est de même du "statut matrimonial " uniquement chez les femmes. Par contre l'âge de l'individu n'est pas significativement associé au degré de stigmatisation et de discrimination chez les deux sexes.

En considérant l'ensemble des cinq attitudes favorables, on note une accentuation de la tolérance à l'égard des $\mathrm{PVVIH}$ en milieu urbain qu'en milieu rural tant chez les femmes $(8,3 \%$ contre $4,3 \%)$ que chez les hommes (18,1\% contre $10,9 \%)$. De même, cet indicateur suit la même tendance selon le niveau d'instruction des répondants des deux sexes. En effet, plus les femmes sont instruites, plus nombreuses, elles expriment des attitudes bienveillantes envers les PVVIH (II,8\% chez les femmes de niveau secondaire ou plus ; II,7\% chez celles qui ont le niveau primaire et $3,6 \%$ chez les non instruites). Les mêmes tendances sont observées chez les hommes mais de manière un peu plus accentuée (respectivement 22,3\%; 12,3\% et $6,2 \%$ ). Par ailleurs, les proportions des individus qui expriment des attitudes bienveillantes augmentent à mesure que le niveau de vie de leur ménage s'améliore. Ces proportions passent de 3,4\% dans les ménages les plus pauvres à $4,4 \%$ dans les ménages de niveau de vie moyen et $8,8 \%$ dans les ménages les plus riches chez les femmes. La tendance est la même chez les hommes $(8,6 \% ; 11,8 \%$ et $19,0 \%$ respectivement). Le degré d'exposition aux médias induit également une attitude de tolérance à l'égard des PVVIH. En effet, les femmes exposées plus d'une fois par semaine aux médias $(8,2 \%$ contre $3,7 \%$ pour celles qui n'y sont jamais exposées) se comporteraient de manière plus tolérante vis-à-vis des PVVIH. II en est de même chez les hommes (I5,8\% contre $8,5 \%$ respectivement). En ce qui concerne la religion, on note que les femmes chrétiennes $(7,4 \%)$, les sans religions $(5,3 \%)$ suivi des musulmanes $(4,7 \%)$ se montreraient plus tolérantes à l'égard des $\mathrm{PVVIH}$. Par contre, chez les hommes, la tendance est plus élevé chez les sans religions $(16,7 \%)$ suivi des chrétiens $(16,2 \%)$ et des musulmans $(10,5 \%)$. L'analyse selon le groupe ethnique révèle que les femmes Yorouba $(8,4 \%)$, Fon $(7,2 \%)$ et Adja (6,3\%) auraient une attitude plus bienveillante à l'égard des $\mathrm{PWVIH}$ alors que chez les hommes, ce sont plutôt les Adja $(21,1 \%)$, les Fon $(15,5 \%)$ et les Yorouba (13,3\%) qui se montrerait plus tolérants. En ce qui concerne l'état matrimonial, les femmes célibataires $(8,5 \%)$ suivi des veuves ou séparées $(7,9 \%)$ se montreraient plus tolérantes http://aps.journals.ac.za 
envers les $\mathrm{PWVIH}$ que les femmes en union $(5,3 \%)$ se montreraient plus tolérantes envers les $\mathrm{PVVIH}$ si elles se trouvaient confronter aux cinq attitudes réunies.

\section{Facteurs associés au niveau de tolérance envers les PVVIH au Bénin}

Les déterminants du niveau de tolérance envers les PVVIH varient selon le sexe des répondants (cf. tableau 4 ci-dessous). Aussi bien chez les femmes que chez les hommes, le niveau d'instruction, l'exposition aux médias, la religion et le groupe ethnique d'appartenance sont les facteurs associés au degré de tolérance à l'égard des $\mathrm{PVVIH}$ au seuil de $5 \%$. En plus, l'état matrimonial, l'activité économique, le niveau de vie du ménage et le milieu de résidence influencent également ce phénomène uniquement chez les femmes.

Les résultats montrent que, le risque relatif pour les femmes résidant en milieu urbain d'exprimer au moins une attitude bienveillante envers les $\mathrm{PVVIH}$ par rapport à aucune attitude bienveillante était 19\% moins élevé que chez celles résidant en milieu rural. De même, le risque pour les femmes vivant dans les ménages de faible et moyen niveaux de vie d'exprimer au moins une attitude bienveillante envers les PVIH par rapport à aucune attitude était respectivement de $33 \%$ et $26 \%$ moins élevé que chez celles vivant dans les ménages riches. S'agissant de toutes les cinq attitudes, le risque est respectivement de $46 \%$ et $41 \%$ moins élevé chez les femmes qui vivent dans les ménages de faible et moyen niveaux de vie que celles vivant dans les ménages riches.

Quel que soit le sexe des répondants, le risque d'exprimer des attitudes bienveillantes à l'égard des PVVIH plutôt que de n'exprimer aucune attitude augmente avec le niveau d'instruction. En effet, le risque pour les femmes qui ont les niveaux primaire et secondaire ou plus d'avoir exprimé au moins l'une quelconque des cinq attitudes bienveillantes envers les $\mathrm{PWVIH}$ par rapport à aucune attitude exprimée, était respectivement $33 \%$ et $296 \%$ plus élevé que chez les femmes sans niveau d'instruction. En considérant, l'ensemble des cinq attitudes bienveillantes, les mêmes tendances s'observent avec des risques relatifs beaucoup plus élevés pour les femmes du niveau primaire $(R R R=91$; $95 \%$ [IC : $\mathrm{I}, 42-2,57])$ et pour celles du niveau secondaire ou plus (RRR=9,97;95\% [IC : 6,88-I4,45]). Chez les hommes la tendance est encore plus accentuée avec une augmentation importante des risques relatifs. Comparés donc au même groupe de référence, le risque relatif pour les hommes du niveau primaire et ceux du niveau secondaire ou plus était respectivement $161 \%$ et $1255 \%$ plus élevé d'exprimer toutes les cinq attitudes bienveillantes envers les $\mathrm{PVVIH}$ par rapport à aucune.

Toutes choses égales par ailleurs, le risque pour les femmes agricultrices d'être tolérantes à l'égard des $\mathrm{PVVIH}$ dans une à quatre situations par rapport à aucune est $27 \%$ moins élevé que chez les femmes n'exerçant aucune activité. De même, les femmes célibataires ont $29 \%$ moins de chance de se montrer tolérantes si elles se trouvaient confrontées aux cinq situations que celles qui sont en union. Par ailleurs, comparées aux femmes chrétiennes, le risque relatif pour les femmes des religions endogènes et celles " sans religion " d'être tolérantes à l'égard des PVIIH dans au moins une attitude par rapport à aucune attitude bienveillante était respectivement $29 \%$ et $27 \%$ moins élevé. Chez les hommes, le risque pour les musulmans et les "sans religion " d'avoir des attitudes tolérantes dans une à quatre situations envers les $\mathrm{PWVIH}$ était respectivement $52 \%$ et $59 \%$ moins élevé que chez les chrétiens.

En considérant le groupe ethnique Adja, le risque pour les femmes Fon, Bariba, Yorouba et autres groupes ethniques d'exprimer toutes les cinq attitudes tolérantes par rapport à aucune attitude, était respectivement $63 \% ; 71 \% ; 61 \%$ et $80 \%$ moins élevé. Chez les hommes, comparés au même groupe de référence, les Fon, Bariba et autres groupes ethniques ont respectivement $79 \% ; 81 \%$ et $69 \%$ moins de chance d'être tolérants s'ils se trouvaient dans les cinq situations. Enfin, le risque pour les femmes qui sont exposées une fois par semaine et de celles qui ne sont pas du tout exposées aux médias d'exprimer les cinq attitudes bienveillantes à l'égard des $\mathrm{PVVIH}$ par rapport à aucune était respectivement de $29 \%$ et $38 \%$ moins élevé que chez celles qui sont exposées plus d'une fois par semaine.

\section{Discussion}

Les analyses établissent un lien positif entre le niveau d'instruction secondaire et plus et l'attitude de bienveillance à l'égard des PVIH. II en est de même pour l'exposition aux médias. Un niveau d'instruction élevé offre plus de capacité et de possibilité de connaissance, de discernement et de s'informer sur la réalité du $\mathrm{VIH} / \mathrm{Sida}$ au sein des personnes infectées et affectées pour mieux comprendre leur état d'esprit. Les travaux de Nzié (2010) au Cameroun confirment que le risque de stigmatisation des $\mathrm{PWVIH}$ diminue avec le niveau d'instruction.

En ce qui concerne les médias on note qu'ils diffusent comme d'autres sources, des informations sur le $\mathrm{VIH}$ dont certaines sont parfois erronées et contribuent à exposer les $\mathrm{PWVIH}$ à la stigmatisation. Face à la mauvaise information, se sentir porteur du virus du Sida est considéré comme un drame d'autant 
plus difficiles à vivre que les raisons de ressentir de la détresse dans une telle situation sont multiples en nature et en intensité. II s'agit a priori du présage d'une mort qui peut parfois dépasser les angoisses qu'engendrerait la maladie elle-même (Macalou et Konaté, 2006 ; Egrot, 2007). Or, comme toute maladie chronique, il est possible de vivre avec le $\mathrm{VIH} /$ Sida pendant longtemps sous certaines conditions thérapeutiques. C'est pourquoi considérer le Sida comme toute maladie chronique devrait être une priorité en termes de communication et d'information pour améliorer la vie des $\mathrm{PWIH}$ (Diarra et Moumouni et al., 2008). Avec la prolifération des médias au Bénin, l'information est de plus en plus accessible et à moindre coût auprès des médias qui diffusent des messages de sensibilisation sur le $\mathrm{VIH} /$ Sida. Dans ces conditions, ne pas s'informer peut entretenir la méconnaissance du $\mathrm{VIH} /$ Sida, pousser à des conduites à risque ou amener à considérer la maladie (Sida) comme une sanction résultant de la transgression des normes sociales du fait de son lien avec la sexualité et au sang qui sont porteurs d'une forte charge symbolique (Desclaux, 2003). II convient cependant de souligner qu'avec l'avènement des Technologies de l'Information et de la Communication (TIC), il est plus difficile de contrôler la qualité des informations sur le $\mathrm{VIH} / \mathrm{Sida}$ diffusées par les médias. Le caractère transnational des nouveaux médias rend encore plus complexe leur régulation ce qui fait d'eux un vecteur qui comporte également des risques.

En ce qui concerne la religion, Novignon et al. (2014) au Ghana ont révélé des liens positifs entre l'appartenance religieuse et la stigmatisation associée au $\mathrm{VIH}$. La tolérance figure parmi les caractéristiques du christianisme, du moins dans sa doctrine de base. Cela peut justifier en partie que l'attitude de bienveillance associée aux PVVIH soit en relation avec le christianisme qui prêche la tolérance à toutes épreuves. L'évangile de Matthieu 5, 38-42 dans la Sainte Bible invite à la nouvelle justice face à la loi du talion. Mais cette posture théologique n'explique pas toujours les interactions entre les $\mathrm{PVVIH}$ et les " autres ». Les travaux de Trinitapoli (20I5, op.cit.) montrent qu'au Malawi, le regard porté par les chefs religieux sur le $\mathrm{VIH} / \mathrm{Sida}$ est fondée sur leur compréhension épidémiologique locale qui privilégie une approche pragmatique visant à raffermir le contrôle religieux, à préserver la stabilité des communautés et à freiner la propagation du $\mathrm{VIH}$. Cette approche plus concentrée sur la communauté que sur l'individu amène l'auteur à considérer la religion plutôt comme une ressource dont les individus peuvent se servir stratégiquement dans la conduite de leur existence.
Les analyses montrent que femmes en union conjugale et celles vivant en milieu rural ont une attitude bienveillante à l'égard des $\mathrm{PVVIH}$. De façon générale, la prévalence au $\mathrm{VIH}$ est plus élevée chez les femmes en âge de procréer que les hommes au Bénin (INSAE et ICF, 20I3, op.cit.). Généralement, les femmes en union ont moins de possibilité de contrôler la sexualité de leur conjoint. La situation est encore plus complexe lorsqu'elles sont dans une union polygamique. Les femmes en union savent donc qu'elles ne sont pas à l'abri d'une infection au $\mathrm{VIH}$ du fait de leur conjoint. Cette crainte peut les amener à comprendre davantage les difficultés des PVIH et les soutenir. De même, vivre en dehors des liens du mariage notamment en milieu rural peut exposer la femme à des discours d'immoralité qui ne relèvent pas moins de la stigmatisation. Le déficit d'autonomie des femmes en union conjugale peut également expliquer une attitude bienveillante de façade à l'égard des $\mathrm{PVVIH}$ mais qui en réalité cache soit une indifférence, soit une intolérance.

Sur un autre plan, contrairement aux résultats de Nzié (2010, op.cit.) et comme le soulignent Novignon et al., (20l4, op.cit.), vivre en milieu rural diminue le risque d'intolérance associé au $\mathrm{VIH}$. La solidarité qui caractérise la vie à la campagne peut pousser les femmes à avoir une attitude bienveillante à l'égard des $\mathrm{PVVIH}$. Bien souvent, la vie en communauté se manifeste par le partage des peines et bonheur des uns par les autres. L'attitude bienveillante envers les PVIH chez les femmes rurales peut participer de l'entraide et de la solidarité. Cela peut également revêtir un soutien psychologique social ou spirituel qui pourrait contribuer à atténuer les émotions notamment, l'angoisse résultant de la découverte de leur séropositivité perçue comme prélude à une mort inéluctable (Zongo et al., 2009).

Les résultats selon lesquels les femmes vivant dans des ménages de niveau de vie élevé ont une attitude bienveillante à l'égard es $\mathrm{PVVIH}$ sont en partie conformes à ceux de l'enquête MICS 2014 au Bénin (INSAE et UNICEF, 20I5) qui ont mis en relief un lien positif entre l'expression d'attitudes bienveillantes à l'égard des $\mathrm{PVVIH}$ et le niveau de vie (3\% dans les ménages les plus pauvres et $13 \%$ dans ceux plus riches pour les femmes contre $9 \%$ et $20 \%$ respectivement pour les hommes). Si Essendi et Wandibba (20I I) au Kenya ont mis en relief une forte stigmatisation associée à la tuberculose et au $\mathrm{VIH} / \mathrm{Sida}$ et à la pauvreté, les travaux de Nzié (2010, Op.cit.) au Cameroun et de Novignon et al., (2014, op.cit.) au Ghana ont révélé l'attitude bienveillante des riches à l'égard des $\mathrm{PWVIH}$.

Les femmes vivant dans les ménages de niveau de vie élevé ont plus de possibilité d'apporter un soutien 
aux personnes en difficulté comme les $\mathrm{PWVIH}$. Leur attitude bienveillante peut annoncer une générosité en cas de nécessité et conforter leur leadership au sein de la société.

En ce qui concerne les femmes n'ayant pas d'activité économique, elles sont le plus souvent dans une situation instable et d'insécurité. Cette vulnérabilité peut les amener à comprendre les difficultés des PVIH car les deux parties partagent des difficultés de nature différente. Ces femmes peuvent même espérer profiter du dynamisme de certaines associations de $\mathrm{PVVIH}$ qui sont des creusets de solidarité, d'entraide et de développement d'activités génératrices de revenu. Ces associations qui représentent une niche de vie pour leurs membres peuvent susciter l'intérêt des personnes n'ayant pas d'activité économique.

En terme d'implication pour les politiques publiques on note que l'accès universel à la maîtrise du $\mathrm{VIH}$ à travers sa prévention et la prise en charge des PVIH ne saurait être une réalité si des séropositifs continuent de faire l'objet de stigmatisation.

Désormais les politiques publiques pourraient prendre en compte le niveau d'instruction, l'exposition aux médias, la religion et le groupe ethnique pour initier des actions dans la population en générale. Par ailleurs, l'état matrimonial, le milieu de résidence, l'activité économique et le niveau de vie du ménage pourraient servir de base pour des actions ciblées à l'endroit des femmes dans les futurs programmes de prise en charge et de protection des $\mathrm{PVVIH}$ contre la stigmatisation liée aux disparités de genre. Pour être efficientes, les nouvelles stratégies de prévention du $\mathrm{VIH}$ et de prise en charge des $\mathrm{PVVIH}$ gagneraient à mobiliser une pédagogie holistique genre-sensible dans leur mise en œuvre. Dans ce sens, les politiques et programmes développés en faveur des PVVIH devront mettre un accent particulier sur les PVVIH les plus vulnérables notamment celles qui sont peu favorisées en terme de disparité de genre. Ces interventions pourraient viser le renforcement du soutien psychologique individuel et de groupe en plus des soins médicaux, de l'appui nutritionnel et d'autres conseils qui leur sont prodigués ainsi qu'aux personnes qui s'occupent de leur prise en charge, tout en soutenant le maintien des enfants à l'école le plus longtemps possible.

\section{Conclusion}

L'objectif de cet article est de déterminer les disparités de genre en matière de stigmatisation des $\mathrm{PVVIH}$. Les analyses montrent que quel que soit le sexe du répondant, avoir un niveau d'instruction élevé, être exposé aux médias plus d'une fois par semaine, être chrétien et appartenir au groupe ethnique Adja déterminent l'attitude bienveillante à l'égard des $\mathrm{PVVIH}$. II en est de même pour les femmes qui sont en union conjugale, celles qui vivent en milieu rural, ou celles vivant dans des ménages de niveau de vie élevé ou celles n'ayant pas activité économique.

En dépit des résultats obtenus, cette étude comporte des limites qu'il convient de souligner. Premièrement, l'indicateur du niveau de stigmatisation et de discrimination à l'égard des PVIH tel qu'il est construit peut être source de biais et pourrait minimiser le phénomène de stigmatisation. En effet, dans la mesure où l'indicateur a été appréhendé à partir des questions sur les perceptions ou les comportements et les attitudes des individus s'ils se trouvaient devant différentes situations avec les $\mathrm{PWVIH}$, ces individus peuvent fournir des réponses qui ne reflètent pas toujours la même attitude s'ils se trouvaient en situation réelle. Deuxièmement, l'étude ne permet pas de connaître l'évolution du phénomène, ni les changements d'attitudes et de comportements des répondants dans le temps. Enfin, l'échantillon ne permet pas de distinguer réellement les séropositifs.

En termes de perspective de recherche, il sera nécessaire de réaliser des études mixtes (combinant données quantitatives et qualitatives) prenant en compte le genre sur les attitudes de stigmatisation à l'égard des $\mathrm{PWVIH}$ en vue d'appréhender le phénomène dans sa globalité.

\section{Références bibliographiques}

Alpe, Y. et al. (2005) "Lexique de sociologie », Paris Dalloz, 329p

Atta, K. (2012) "Approche psycho-qualitative du $\mathrm{VIH} /$ Sida en contexte africain : cas du vécu de l'infection chez des patients du Service des maladies infectieuses et tropicales (SMIT) d'Abidjan " Recherches qualitatives - Vol. $3 \mathrm{I}(\mathrm{I})$, pp. |30-I5|. http://www.recherchequalitative.qc.ca/ Association pour la recherche qualitative

Beaulieu, M. (20I4) «Attitudes stigmatisantes envers les personnes vivant avec le $\mathrm{VIH}$ : développement et validation d'un instrument de mesure intégrant un dispositif participatif ", Thèse de doctorat $(\mathrm{PhD})$ en Santé publique Option Promotion de la santé, Faculté des études supérieures, Département de médecine sociale et préventive, École de santé publique, 356p.

Benin (République du), (1990) «Loi N 90-32 du II décembre 1990 portant Constitution de la République du Bénin », Cotonou, 37p.

Benin (République du) (2006) « Loi No 2005-3I du 10 avril 2006 portant prévention, prise en charge 
et contrôle du VIH/SIDA en République du Bénin ", Cotonou, 27p.

Benoist, J., (2007), "Logique de la stigmatisation, éthique de la déstigmatisation ", Revue L'Information psychiatrique, vol. 83, no 8, pp. 649-654.

Bruyn, T (1998) « HIV/AIDS and discrimination: a discussion paper ", Canadian HIV/AIDS Legal Network and Canadian AIDS Society, Montréal, 235p.

CNLS, 2016 "Rapport de suivi de la déclaration de politique sur le VIH/Sida au Bénin ", Cotonou, $61 \mathrm{p}$.

Desclaux, A. (200I) «Une étrange absence de crise : l'adaptation des systèmes de santé du Sud au $\mathrm{VIH} / \mathrm{SIDA}$ ». In: Hours B, editor. Systèmes et politiques de santé. De la santé publique à l'anthropologie, Paris, Karthala ; pp 87- 102.

Desclaux, A. (2003) " VIH/SIDA, stigmatisation et discrimination : une approche anthropologique ". Etudes et rapports, série spéciale $n^{\circ} 20$, Division des politiques culturelles et du dialogue interculturel, UNESCO 2003, pp. I-I0.

Desclaux, A. et TAVERNE, B. (2000), Allaitement et $\mathrm{VIH}$ en Afrique de l'Ouest : de l'anthropologie à la santé publique, Paris, Les Éditions Karthala, Collection Médecines du monde, 556p.

Diarra, A. et Moumounl, A. et al. (2008) "Le vécu quotidien des personnes vivant avec le $\mathrm{VIH}$ à Niamey » Niamey, Etudes et travaux du LASDEL, $50 p$

Egrot, M. (2007), "Renaître d'une mort sociale annoncée : recomposition du lien social des personnes vivant avec le $\mathrm{VIH}$ en Afrique de |'Ouest (Burkina Faso, Sénégal) », Cultures \& sociétés, pp.49-56.

Essendi $\mathrm{H}$, et Wandibba S. (20ll) : "Barriers to health care by women infected with Tuberculosis in Kibera slums in Nairobi”, Kenya, African Population Studies Vol 25, I (Supplement) 20I I, pp 17-33.

Goffman, E. (1977) Stigmates : les usages sociaux des handicaps, Paris, Ed. de Minuit, 1977 (lère éd. 1963), Collection Le sens commun , 176p

INSAE et ICF International (20I3), "Enquête Démographique et de Santé du Bénin 20II-20I2 ». Calverton, Maryland, USA, Rapport final, $55 \mathrm{Ip}$.

INSAE et UNICEF (20I5) «Enquête par grappes à indicateurs multiples 2014 », Rapport final, Cotonou, 247p.

Konan, Y., E. \& al. (2008) : "Perception des personnes vivant avec le $\mathrm{VIH}$ sur l'infection à $\mathrm{VIH}$. À propos d'une enquête réalisée au Centre d'assistance socio-médicale de Treichville, Abidjan, Côte d'Ivoire», Cah. Santé Publique, Vol. 7, n`I- 2008 EDUCl, pp7-I6.
Ky-Zerbo, O. et al. (20|4) "La stigmatisation des PVIH en Afrique: analyse de ses formes et manifestations au Burkina Faso " NIH Public Access Sante Publique ; 26(3): 375-384.

Le Breton, D. (2012). L'interactionnisme symbolique, Paris, PUF, 256p.

Macalou, C. et Konate, S. (2006) "Lutte contre la stigmatisation et la discrimination des personnes vivant avec le $\mathrm{VIH} / \mathrm{SIDA}$ au moyen de l'éducation non formelle dans les communes de Koulikoro et de Banamba en République du Mali », ROCARE, 29p.

Ministère de la Santé, (2018) : "Annuaire des statistiques sanitaires 2017 », Cotonou, I43p.

Novignon, J. \& al., (2014) "HIV/AIDS-related stigma and HIV test uptake in Ghana: evidence from the 2008 Demographic and Health Survey", African Population Studies, Vol. 28, N ${ }^{\circ} 3,2014$.

Nzie, A. (2010), "Facteurs associés aux attitudes de stigmatisation à l'égard des personnes vivant avec le VIH/SIDA au Cameroun ", Mémoire de Master Professionnel en Démographie, IFORD, Yaoundé, 103p.

ONUSIDA (2002), " les jeunes et le VIH/Sida : une solution à la crise, Genève, ONUSIDA, 32p.

ONUSIDA (2003) "Conseils et test VIH volontaire : une voie d'accès à la prévention et aux soins. Genève: Collection meilleures pratiques de I'ONUSIDA. 65p

ONUSIDA (2005) «Stigmatisation, discrimination et violations des droits de l'homme associées au $\mathrm{VIH}$. Etudes de cas des interventions réussies ", Genève, collection meilleures pratiques de I'ONUSIDA, 80p.

ONUSIDA (2015) "Statistiques mondiales, Journée mondiale de lutte contre le Sida 2015»ww.unaids.org/en/resources/campaigns /HowAIDSchangedeverything/factsheet

PNLS (2004) «Politique, normes et procédures pour la prise en charge des personnes vivant avec le $\mathrm{VIH}$ au Bénin »Cotonou. I37p.

RAP+ Afrique de l'Ouest et du Centre et ONUSIDA (2002) : " Analyse situationnelle de la discrimination et stigmatisation envers les Personnes Vivant avec le $\mathrm{VIH} / \mathrm{SIDA}$ en Afrique de l'Ouest et du Centre : considérations éthiques et juridiques ", 42p

$\mathrm{RNP}+(20 / 2)$ « VIH Leadership à travers la responsabilisation: The people living with HIV Stigma Index », Dakar, 82p.

Zongo, S. et Ouattara, F (2008) : "Quand la prévention de la transmission mère-enfant du $\mathrm{VIH}$ " cause problèmes ": une ethnographie des relations interprofessionnelles dans trois sites de Ouagadougou ", Spécial hors-série $\mathrm{n}^{\circ} \mathrm{I}$, Science et technique, Sciences de la santé, pp I07-I I5.

http://aps.journals.ac.za 
Skinner, D. et Mfecane, S. (2004) "Stigma, discrimination and the implications for people living with HIV/AIDS in South Africa ", Journal des Aspects Sociaux du VIH/SIDA, Vol. I n³, pp I57164.

Trinitapoli, J. (2015) «Sida et vie religieuse au Malawi : repenser l'influence de la dynamique démographique sur les comportements culturels » Paris, Population, INED Vol. 70, pp 265-293.

Zongo, M. et al. (2009) «Prise en charge psychosociale des personnes vivant avec le $\mathrm{VIH}$ au Bénin ", SFSP.| "Santé Publique "Vol. 2I, pp 63I-639.

Tableau I : Répartition des femmes et des hommes selon les caractéristiques de l'échantillon

\begin{tabular}{|c|c|c|c|c|}
\hline \multirow[b]{2}{*}{ Variables/modalités } & \multicolumn{2}{|l|}{ Femmes } & \multicolumn{2}{|l|}{ Hommes } \\
\hline & Effectifs & $\begin{array}{l}\text { Proportion } \\
\text { (\%) }\end{array}$ & Effectifs & $\begin{array}{l}\text { Proportion } \\
(\%)\end{array}$ \\
\hline Niveau de vie du ménage & 14849 & 100 & 4813 & 100 \\
\hline Pauvre & 4777 & 32,2 & 1518 & 31,5 \\
\hline Moyen & 2804 & 18,9 & 925 & 19,2 \\
\hline Riche & 7268 & 48,9 & 2370 & 49,2 \\
\hline Milieu de résidence & 14849 & 100 & 4813 & 100 \\
\hline Urbain & 7108 & 47,9 & 2255 & 46,9 \\
\hline Rural & $774 \mid$ & 52,1 & 2558 & 53,1 \\
\hline Religion & 14849 & 100 & 4813 & 100 \\
\hline Chrétien & 8982 & 60,5 & 2700 & 56,1 \\
\hline Musulman & 2998 & 20,2 & 1069 & 22,2 \\
\hline Endogène & 1921 & 12,9 & 774 & 16,1 \\
\hline Sans religion & 948 & 6,4 & $27 \mid$ & 5,6 \\
\hline Ethnie & 14849 & 100 & 4813 & 100 \\
\hline Adja & 2418 & 16,3 & 710 & 14,7 \\
\hline Fon & 7162 & 48,2 & 2363 & 49,1 \\
\hline Bariba & 1133 & 7,6 & 405 & 8,4 \\
\hline Yorouba & 1768 & 11,9 & 572 & 11,9 \\
\hline Autres ethnies & 2368 & 16,0 & 764 & 15,9 \\
\hline Niveau d'instruction & 14849 & 100 & 4813 & 100 \\
\hline Aucun niveau & 8407 & 56,6 & 1658 & 34,5 \\
\hline Niveau primaire & 2733 & 18,4 & 1167 & 24,2 \\
\hline Secondaire ou plus & 3709 & 25,0 & 1988 & 41,3 \\
\hline Activité économique & 14085 & 94,90 & 4772 & 99,20 \\
\hline Aucune & 5297 & 35,7 & 1178 & 24,5 \\
\hline Cadre & 3072 & 20,7 & 899 & 18,7 \\
\hline Commerçant & 2616 & 17,6 & 206 & 4,3 \\
\hline Agriculteur & 2073 & 14 & 1432 & 29,7 \\
\hline Autre & 1027 & 6,9 & 1057 & 22,0 \\
\hline Age de la femme & | 4849 & 100 & 4813 & 100 \\
\hline I5-24 ans & 5187 & 34,9 & 1507 & 31,3 \\
\hline $25-34$ ans & 5243 & 35,3 & 1214 & 25,2 \\
\hline $35-49$ ans & 4419 & 29,8 & 1429 & 29,7 \\
\hline $50-64$ ans & - & - & 664 & 13,8 \\
\hline Exposition aux médias & 14849 & 100 & 4813 & 100 \\
\hline N’a jamais été exposée & 4894 & 33 & 750 & 15,6 \\
\hline Exposée une fois par semaine & 2995 & 20,2 & 1023 & 21,2 \\
\hline $\begin{array}{l}\text { Exposée plus d'une fois par } \\
\text { semaine }\end{array}$ & 6960 & 46,9 & 3040 & 63,2 \\
\hline État matrimonial & 14849 & 100 & 4813 & 100 \\
\hline Célibataire & 3620 & 24,4 & 1684 & 35 \\
\hline En union & 10377 & 69,9 & 2980 & 61,9 \\
\hline En rupture d'union & 852 & 5,7 & 149 & 3,1 \\
\hline
\end{tabular}

Source : Enquête Démographique et de Santé du Bénin, 2011-2012 
Tableau 2 : Pourcentage des hommes et femmes de 15-49 ans ayant entendu parler du Sida et exprimant des attitudes de tolérance dans des situations déterminées à l'égard des PVVIH

\begin{tabular}{|c|c|c|c|c|}
\hline \multirow[t]{2}{*}{ Attitudes de tolérance à l'égard des PVVIH } & \multicolumn{2}{|l|}{ Femmes } & \multicolumn{2}{|l|}{ Hommes } \\
\hline & Effectifs & $\%$ & Effectifs & $\%$ \\
\hline S'occuper à la maison d'un parent ayant le sida & 8579 & 57,8 & 3381 & 70,2 \\
\hline Acheter des légumes frais à un commerçant vivant avec le $\mathrm{VIH}$ & 5995 & 40,4 & 2126 & 44,2 \\
\hline $\begin{array}{l}\text { Penser qu'une enseignante vivant avec le VIH mais qui n'est pas } \\
\text { malade devrait être autorisée à continuer d'enseigner }\end{array}$ & 8069 & 54,3 & 2818 & 58,5 \\
\hline $\begin{array}{l}\text { Penser qu'il n'est pas nécessaire de garder secret l'état d'un membre } \\
\text { de la famille ayant le VIH }\end{array}$ & 6180 & $4 I, 6$ & 2514 & 52,2 \\
\hline $\begin{array}{l}\text { Penser qu'on peut partager la nourriture avec une PVIH sans } \\
\text { contracter le VIH }\end{array}$ & 10056 & 67,7 & 3703 & 76,9 \\
\hline
\end{tabular}

Source : Enquête Démographique et de Santé du Bénin, 20I I-2012

Graphique I : la proportion des femmes et d'hommes exprimant des attitudes bienveillantes sur tous les cinq indicateurs à l'égard des personnes vivant avec le VIH

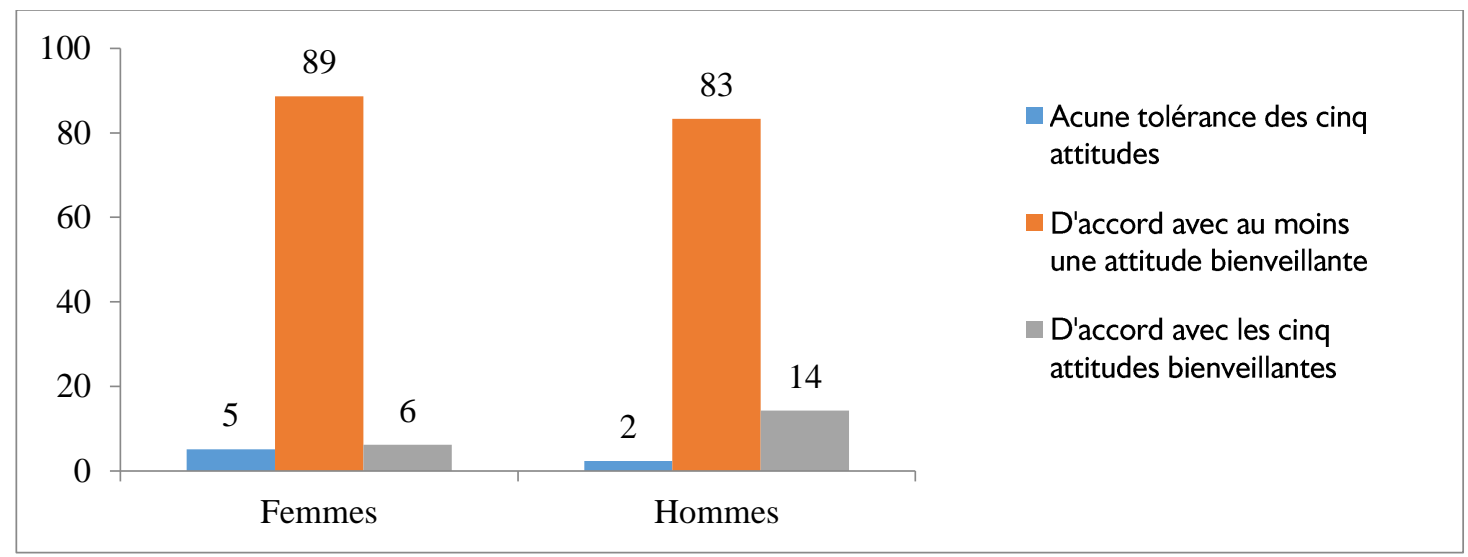

Source : Enquête Démographique et de Santé du Bénin, 20I I-2012 
Tableau 3 : Répartition (\%) des répondants exprimant le degré de tolérance par sexe selon certaines caractéristiques

\begin{tabular}{|c|c|c|c|c|c|c|c|c|c|c|c|c|c|c|c|c|c|c|}
\hline \multirow{3}{*}{ Variables } & \multicolumn{9}{|c|}{ Nombre d'attitudes favorables chez les femmes } & \multicolumn{9}{|c|}{ Nombre d'attitudes favorables chez les hommes } \\
\hline & \multicolumn{3}{|c|}{ Aucun } & \multicolumn{3}{|c|}{ I à 4 attitudes } & \multicolumn{3}{|c|}{5 attitudes } & \multicolumn{3}{|c|}{ Aucun } & \multicolumn{3}{|c|}{ I à 4 attitudes } & \multicolumn{3}{|c|}{5 attitudes } \\
\hline & Eff & $\%$ & IC (95\%) & Eff & $\%$ & IC (95\%) & Eff & $\%$ & IC (95\%) & Eff & $\%$ & IC (95\%) & Eff & $\%$ & IC (95\%) & Eff & $\%$ & IC (95\%) \\
\hline \multicolumn{19}{|c|}{ Niveau de vie du ménage } \\
\hline Pauvre & 348 & 7,3 & $(6,5-8,0)$ & 4267 & 89,3 & $(88,5-90,2)$ & 162 & 3,4 & $(2,9-3,9)$ & 51 & 3,4 & $(2,5-4,3)$ & 1337 & 88,1 & $(86,4-89,7)$ & 130 & 8,6 & $(7, I-I 0)$ \\
\hline Moyen & 158 & 5,6 & $(4,7-6,5)$ & 2524 & 90 & $(88,9-91,1)$ & 122 & 4,4 & $(3,6-5,1)$ & 31 & 3,4 & $(2,2-4,5)$ & 785 & 84,9 & $(82,6-87,2)$ & 109 & 11,8 & $(9,7-\mid 3,9)$ \\
\hline Riche & 259 & 3,6 & $(3, I-4,0)$ & 6368 & 87,6 & $(86,9-88,4)$ & $64 I$ & 8,8 & $(8,2-9,3)$ & 34 & $\mathrm{I}, 4$ & $(I, 0-I, 9)$ & 1886 & 79,6 & $(77,9-8 I, 2)$ & 450 & 19 & $(17,4-20,6)$ \\
\hline \multicolumn{19}{|l|}{ Milieu de résidence } \\
\hline Urbain & 316 & 4,4 & $(4,0-4,9)$ & 6199 & 87,2 & $(86,4-88,0)$ & 592 & 8,3 & $(7,7-9,0)$ & 36 & 1,6 & $(I, I-2, I)$ & $|8| \mid$ & 80,3 & $(78,6-81,9)$ & 409 & 18,1 & $(16,5-19,7)$ \\
\hline Rural & 449 & 5,8 & $(5,3-6,3)$ & 6960 & 89,9 & $(89,2-90,3)$ & 333 & 4,3 & $(3,8-4,7)$ & 81 & 3,2 & $(2,5-3,8)$ & 2197 & 85,9 & $(84,5-87,3)$ & 280 & 10,9 & $(9,7-12,2)$ \\
\hline \multicolumn{19}{|l|}{ Religion } \\
\hline Chrétien & 365 & 4,1 & $(3,6-4,5)$ & 7949 & 88,5 & $(87,8-89,2)$ & 668 & 7,4 & $(6,9-8,0)$ & 44 & 1,6 & $(I, 2-2, I)$ & 2219 & 82,2 & $(80,7-83,6)$ & 437 & 16,2 & $(14,8-17,6)$ \\
\hline Musulman & 205 & 6,8 & $(5,9-7,8)$ & 2652 & 88,5 & $(87,3-89,6)$ & 140 & 4,7 & $(3,9-5,4)$ & 35 & 3,3 & $(2,2-4,4)$ & 922 & 86,2 & $(84,2-88,3)$ & 112 & 10,5 & $(8,6-I 2,3)$ \\
\hline Endogène & 127 & 6,6 & $(5,5-7,7)$ & 1727 & 89,9 & $(88,6-91,3)$ & 67 & 3,5 & $(2,7-4,3)$ & 25 & 3,2 & $(2,0-4,5)$ & 653 & 84,5 & $(81,9-87,0)$ & 95 & 12,3 & $(10,0-4,6)$ \\
\hline Sans religion & 67 & 7,1 & $(5,5-8,8)$ & 831 & 87,7 & $(85,5-89,7)$ & 50 & 5,3 & $(3,9-6,7)$ & II & 4,1 & $(I, 8-6,7)$ & 214 & 79,3 & $(74,3-84,0)$ & 45 & 16,7 & $(|2|-2 \mid, 0)$, \\
\hline \multicolumn{19}{|l|}{ Ethnie } \\
\hline Adja & 56 & 2,3 & $(1,7-2,9)$ & 2209 & 91,4 & $(90,2-92,5)$ & 153 & 6,3 & $(5,4-7,3)$ & 7 & 1 & $(0,3-I, 8)$ & 553 & 77,9 & $(74,8-80,9)$ & 150 & 21,1 & $(18, \mid-24, I)$ \\
\hline Fon & 347 & 4,8 & $(4,3-5,4)$ & 6300 & 88 & $(87,2-88,7)$ & 514 & 7,2 & $(6,6-7,8)$ & 66 & 2,8 & $(2, I-3,5)$ & 1931 & $8 \mathrm{I}, 7$ & $(80,2-83,3)$ & 366 & 15,5 & $(14,0-16,9)$ \\
\hline Bariba & 67 & 5,9 & $(4,5-7,3)$ & 1030 & 91 & $(89,3-92,6)$ & 35 & 3,1 & $(2, \mid-4, I)$ & 17 & 4,2 & $(2,3-6,2)$ & 353 & 87,2 & $(83,9-90,5)$ & 35 & 8,6 & $(5,8-I I, 3)$ \\
\hline Yorouba & 103 & 5,8 & $(4,7-6,9)$ & 1517 & 85,8 & $(84,2-87,4)$ & 149 & 8,4 & $(7, I-9,7)$ & 6 & $\mathrm{I}, 6$ & $(0,2-1,9)$ & 490 & 85,7 & $(82,8-88,6)$ & 76 & 13,3 & $(10,5-16, I)$ \\
\hline Autres ethnies & 192 & 8,1 & $(7,0-9,2)$ & 2103 & 88,8 & $(87,5-90,1)$ & 74 & 3,1 & $(2,4-3,8)$ & 20 & 2,6 & $(1,5-3,8)$ & 681 & 89,1 & $(86,9-91,3)$ & 63 & 8,2 & $(6,3-10,2)$ \\
\hline \multicolumn{19}{|l|}{ Niveau d'instruction } \\
\hline Aucun niveau & 576 & 6,9 & $(6,3-7,4)$ & 7530 & 89,6 & $(88,9-90,2)$ & 301 & 3,6 & $(3,2-4,0)$ & 69 & 4,2 & $(3,2-5, I)$ & | 487 & 89,7 & $(88,2-91,1)$ & 102 & 6,2 & $(5,01-7,32)$ \\
\hline Niveau primaire & 127 & 4,6 & $(3,8-5,4)$ & 2420 & 88,5 & $(87,3-89,7)$ & 437 & $\mathrm{II}, 7$ & $(5,9-7,8)$ & 31 & 2,7 & $(1,7-3,6)$ & 991 & 85 & $(82,9-87,0)$ & 144 & 12,3 & $(|0,5-| 4,2)$ \\
\hline Secondaire ou plus & 62 & $\mathrm{I}, 7$ & $(I, 3-2, I)$ & 3210 & 86,5 & $(85,4-87,6)$ & 437 & $1 \mathrm{I}, 8$ & $(10,8-12,8)$ & 16 & 0,8 & $(0,4-I, 2)$ & 1530 & 76,9 & $(75, I-78,8)$ & 443 & 22,3 & $(20,4-24, I)$ \\
\hline
\end{tabular}


African Population Studies Vol.33, No. I, Feb. 2019

\begin{tabular}{|c|c|c|c|c|c|c|c|c|c|c|c|c|c|c|c|c|c|c|}
\hline \multirow[t]{2}{*}{ Variables } & \multicolumn{9}{|c|}{ Nombre d'attitudes favorables chez les femmes } & \multicolumn{9}{|c|}{ Nombre d'attitudes favorables chez les hommes } \\
\hline & \multicolumn{3}{|c|}{ Aucun } & \multicolumn{3}{|c|}{ I à 4 attitudes } & \multicolumn{3}{|c|}{5 attitudes } & \multicolumn{3}{|c|}{ Aucun } & \multicolumn{3}{|c|}{ I à 4 attitudes } & \multicolumn{3}{|c|}{5 attitudes } \\
\hline Aucune & 241 & 4,5 & $(4,0-5,1)$ & 2673 & 87 & $(88,4-90,0)$ & 257 & 8,4 & $(5,57-6,87)$ & 18 & $\mathrm{I}, 5$ & $(0,8-2,2)$ & 961 & 81,6 & $(79,3-83,8)$ & 199 & 16,9 & $(14,8-19,1)$ \\
\hline Cadre & 142 & 4,6 & $(3,9-5,4)$ & 2673 & 87 & $(85,8-88,2)$ & 257 & 8,4 & $(7,38-9,34)$ & 13 & $\mathrm{I}, 4$ & $(0,7-2,2)$ & 675 & 75,1 & $(72,3-78,0)$ & 211 & 23,5 & $(20,7-26,2)$ \\
\hline Commerçant & 130 & 5 & $(4,2-5,8)$ & 2309 & 88,3 & $(87, I-89,5)$ & 176 & 6,7 & $(5,76-7,68)$ & 5 & 2,4 & $(0,2-4,3)$ & 173 & 84 & $(79, I-89, I)$ & 28 & 13,6 & $(8,9-18,3)$ \\
\hline Agriculteur & 170 & 8,2 & $(7,0-9,4)$ & 1845 & 89 & $(87,7-90,4)$ & 58 & 2,79 & $(2,08-3,50)$ & 40 & 2,8 & $(1,9-3,6)$ & 1288 & 89,9 & $(88,4-9 \mid, 5)$ & 104 & 7,3 & $(5,9-8,6)$ \\
\hline Autre & 45 & 4,4 & $(3,2-5,7)$ & 920 & 89,6 & $(87,7-9 \mid, 4)$ & 62 & 6 & $(4,57-7,49)$ & 41 & 3,9 & $(2,7-5, I)$ & 883 & 83,5 & $(81,3-85,8)$ & 133 & 12,6 & $(10,6-14,6)$ \\
\hline \multicolumn{19}{|l|}{ Age de l'individu } \\
\hline $15-24$ ans & 246 & 4,7 & $(4,2-5,3)$ & 4589 & 88,5 & $(87,6-89,3)$ & 352 & 6,8 & $(6,10-7,47)$ & 35 & 2,3 & $(1,6-3,1)$ & 1255 & 83,3 & $(8 I, 4-85,2)$ & 216 & 14,3 & $(12,6-16,1)$ \\
\hline $25-34$ ans & 277 & 5,3 & $(4,7-5,9)$ & 4636 & 88,4 & $(87,5-89,3)$ & 330 & 6,3 & $(5,64-6,95)$ & 23 & 1,9 & $(1,2-2,7)$ & 1024 & 84,3 & $(82,3-86,4)$ & 167 & 13,8 & $(I I, 8-\mid 5,7)$ \\
\hline $35-49$ ans & 241 & 5,5 & $(4,8-6,1)$ & 3935 & 89 & $(88, I-90,0)$ & 243 & 5,5 & $(4,83-6,18)$ & 34 & 2,4 & $(1,6-3, I)$ & 1195 & 83,6 & $(81,8-85,6)$ & 200 & 14 & $(12,2-15,8)$ \\
\hline $50-64$ ans & - & - & - & - & - & - & - & - & - & 24 & 3,6 & $(2,2-5,0)$ & 534 & 80,4 & $(77,4-83,4)$ & 106 & 16 & $(13,2-18,8)$ \\
\hline \multicolumn{19}{|l|}{ Exposition aux médias } \\
\hline N’a jamais été exposée & 312 & 6,4 & $(5,7-7, I)$ & 4399 & 89,9 & $(89,0-90,7)$ & 183 & 3,7 & $(3,2 \mid-4,27)$ & 23 & 3,1 & $(1,9-4,3)$ & 663 & 88,4 & $(86,0-90,6)$ & 64 & 8,5 & $(6,6-10,6)$ \\
\hline Une fois par semaine & 180 & 6 & $(5,2-6,9)$ & 2644 & 88,3 & $(87,1-89,4)$ & 171 & 5,7 & $(4,89-6,55)$ & 17 & $\mathrm{I}, 7$ & $(0,9-2,4)$ & 863 & 84,4 & $(82,1-86,6)$ & 143 & 14 & $(11,9-16,1)$ \\
\hline Plus d'une fois par semaine & 273 & 3,9 & $(3,5-4,4)$ & 6117 & 87,9 & $(87,1-88,6)$ & 571 & 8,2 & $(7,56-8,85)$ & 76 & 2,5 & $(I, 9-3,1)$ & 2483 & 81,7 & $(80,3-83,0)$ & 481 & 15,8 & $(14,5-17, I)$ \\
\hline \multicolumn{19}{|l|}{ État matrimonial } \\
\hline Célibataire & 154 & 4,3 & $(3,6-4,9)$ & 3158 & 87,3 & $(86,2-88,3)$ & 307 & 8,5 & $(7,58-9,40)$ & 36 & 2,1 & $(1,4-2,8)$ & $|40|$ & 83,2 & $(81,4-85,0)$ & 247 & 14,7 & $(13,0-16,4)$ \\
\hline En union & 574 & 5,5 & $(5, I-6,0)$ & 9252 & 89,2 & $(88,6-89,8)$ & 551 & 5,3 & $(4,88-5,74)$ & 77 & 2,6 & $(2,0-3,2)$ & 2481 & 83,2 & $(81,9-84,6)$ & 423 & 14,2 & $(12,9-15,4)$ \\
\hline En rupture d'union & 36 & 4,2 & $(2,9-5,5)$ & 749 & 87,9 & $(85,7-90, I)$ & 67 & 7,9 & $(6,05-9,67)$ & 4 & 2,7 & $(0,0-5, I)$ & 126 & 84,6 & $(78,7-90,4)$ & 19 & 12,8 & $(7,5-18,3)$ \\
\hline
\end{tabular}

Source : Enquête Démographique et de Santé du Bénin, 201 I-2012 
Tableau 4 : Résultats des régressions logistiques multinomiales sur le degré de tolérance envers les PVVIH au Bénin

\begin{tabular}{|c|c|c|c|c|c|c|c|c|}
\hline \multirow[t]{3}{*}{ Variables } & \multicolumn{4}{|c|}{ Nombre d'attitudes favorables chez les femmes } & \multicolumn{4}{|c|}{ Nombre d'attitudes favorables chez les hommes } \\
\hline & \multicolumn{2}{|c|}{ Aucune vs I à 4 décisions } & \multicolumn{2}{|c|}{ Aucune vs 5 décisions } & \multicolumn{2}{|c|}{ Aucune vs I à 4} & \multicolumn{2}{|c|}{ Aucune vs 5 décisions } \\
\hline & RRR & $(95 \%$ IC) & RRR & $(95 \%$ IC) & RRR & $(95 \% \mathrm{IC})$ & RRR & $(95 \%$ IC) \\
\hline \multicolumn{9}{|l|}{ Milieu de résidence } \\
\hline Urbain & $0,8 I^{* * *}$ & $(0,67-0,97)$ & 0,91 & $(0,70-I, 40)$ & 1,22 & $(0,76-I, 96)$ & 1,30 & $(0,78-2,18)$ \\
\hline \multicolumn{8}{|l|}{ Niveau de vie du ménage } & Réf \\
\hline Pauvre & 0,67 **** & $(0,54-0,85)$ & 0,54 **** & $(0,39-0,75)$ & 0,70 & $(0,40-I, 24)$ & $0,59 *$ & $(0,3 I-I, I 0)$ \\
\hline Moyen & $0,74^{* * *}$ & $(0,58-0,94)$ & $0,59 * * *$ & $(0,42-0,75)$ & 0,60 & $(0,34-1,06)$ & $0,54 *$ & $(0,29-1,00)$ \\
\hline Riche & Réf & Réf & Réf & Réf & Réf & Réf & Réf & Réf \\
\hline \multicolumn{9}{|l|}{ Religion } \\
\hline Chrétien & Réf & Réf & Réf & Réf & Réf & Réf & Réf & Réf \\
\hline Musulman & 0,80 & $(0,63-I, 0 I)$ & 0,74 & $(0,53-1,03)$ & $0,48 * *$ & $(0,25-0,94)$ & 0,58 & $(0,28-1,19)$ \\
\hline Endogène & $0,7 \mid$ I**** & $(0,56-0,89)$ & $0,56^{* * * *}$ & $(0,39-0,80)$ & 0,60 & $(0,35-1,03)$ & $0,7 \mathrm{I}$ & $(0,39-1,27)$ \\
\hline Sa religion & $0,73^{* *}$ & $(0,55-0,97)$ & 0,77 & $(0,5 I-I, I 7)$ & $0,41 * *$ & $(0,20-0,81)$ & 0,50 & $(0,24-1,06)$ \\
\hline \multicolumn{9}{|l|}{ Ethnie } \\
\hline Adja & Réf & Réf & Réf & Réf & Réf & Réf & Réf & Réf \\
\hline Fon & $0,38^{* * * *}$ & $(0,28-0,5 \mathrm{I})$ & $0,37 * * * *$ & $(0,26-0,53)$ & $0,32 * * *$ & $(0,14-0,70)$ & $0,21 * * *$ & $(0,09-0,47)$ \\
\hline Bariba & $0,46^{* * * *}$ & $(0,31-0,70)$ & $0,29 * * * *$ & $(0,16-0,52)$ & 0,38 & $(0,13-1,10)$ & $0,19 * * *$ & $(0,06-0,59)$ \\
\hline Yorouba & $0,33^{* * * *}$ & $(0,23-0,47)$ & $0,39 * * * *$ & $(0,25-0,60)$ & $\mathrm{I}, 0 \mathrm{I}$ & $(0,32-3,2)$ & 0,56 & $(0,17-1,84)$ \\
\hline Autres ethnies & 0,31 **** & $(0,22-0,44)$ & 0,20 ***** & $(0,13-0,32)$ & 0,62 & $(0,23-1,67)$ & $0,31 * *$ & $(0,11-0,87)$ \\
\hline \multicolumn{9}{|l|}{ Niveau d'instruction } \\
\hline Aucun niveau & Réf & Réf & Réf & Réf & Réf & Réf & Réf & Réf \\
\hline Niveau primaire & I,33** & $(1,06-1,66)$ & I,9|***** & $(1,42-2,57)$ & $\mathrm{I}, 47$ & $(0,91-2,36)$ & $2,61 * * *$ & $(I, 5 I-4,49)$ \\
\hline Secondaire ou plus & $3,96 * * *$ & $(2,88-5,45)$ & $9,97 * * *$ & $(6,88-14,45)$ & $3,85 * * *$ & $(1,96-7,55)$ & $13,55^{* * *}$ & $(6,58-27,90)$ \\
\hline \multicolumn{9}{|l|}{ Activité économique } \\
\hline Aucune & Réf & Réf & Réf & Réf & Réf & Réf & Réf & Réf \\
\hline Cadre & 0,91 & $(0,72-I, I 4)$ & $\mathrm{I}, 34$ & $(I, 00-1,79)$ & $\mathrm{I}, 07$ & $(0,49-2,35)$ & $\mathrm{I}, 35$ & $(0,59-3,07)$ \\
\hline Commerçant & 0,94 & $(0,74-1,19)$ & $\mathrm{I}, 3 \mathrm{I}$ & $(0,95-1,79)$ & 0,82 & $(0,27-2,45)$ & 0,61 & $(0,19-1,97)$ \\
\hline Agriculteur & $0,73^{* * * *}$ & $(0,58-0,91)$ & 0,70 & $(0,48-1,03)$ & $\mathrm{I}, 50$ & $(0,76-2,95)$ & 1,05 & $(0,50-2,22)$ \\
\hline Autre & 1,09 & $(0,78-1,53)$ & 1,30 & $(0,84-2,01)$ & 0,62 & $(0,33-1,18)$ & $0,53 *$ & $(0,27-1,06)$ \\
\hline \multicolumn{9}{|l|}{ Age des individus } \\
\hline I5-24 ans & 0,98 & $(0,79-1,22)$ & 0,83 & $(0,62-1,12)$ & 0,55 & $(0,27-I, I 2)$ & $0,48^{*}$ & $(0,22-1,03)$ \\
\hline $25-34$ ans & Réf & Réf & Réf & Réf & Réf & Réf & Réf & Réf \\
\hline $35-49$ ans & 1,00 & $(0,93-I, 20)$ & 0,93 & $(0,72-I, 20)$ & 0,80 & $(0,46-I, 40)$ & 0,89 & $(0,49-1,62)$ \\
\hline $50-64$ ans & - & - & - & - & 0,57 & $(0,3 I-I, 04)$ & 0,92 & $0,48-I, 80)$ \\
\hline
\end{tabular}


African Population Studies Vol.33, No. I, Feb. 2019

\begin{tabular}{|c|c|c|c|c|c|c|c|c|}
\hline \multicolumn{9}{|l|}{ Exposition aux médias } \\
\hline Non exposée & 0,90 & $(0,75-I, 09)$ & $0,62 * * * *$ & $(0,48-0,80)$ & $\mathrm{I}, 05$ & $(0,64-I, 72)$ & 0,77 & $(0,44-I, 36)$ \\
\hline I fois par semaine & 0,83 & $(0,68-1,02)$ & $0,7 I^{* * *}$ & $(0,54-0,93)$ & $\mathrm{I}, 73^{* * *}$ & $(1,00-2,99)$ & $\mathrm{I}, 7 I^{*}$ & $(0,96-3,05)$ \\
\hline $\begin{array}{l}\text { Plus d'une fois par semaine } \\
\text { État matrimonial }\end{array}$ & Réf & Réf & Réf & Réf & Réf & Réf & Réf & Réf \\
\hline Célibataire & $0,63 * * *$ & $(0,49-0,8 \mathrm{I})$ & $0,7 \mid$ I** & $(0,52-0,99)$ & 0,97 & $(0,48-1,96)$ & 0,82 & $(0,38-1,75)$ \\
\hline En union & Réf & Réf & Réf & Réf & Réf & Réf & Réf & Réf \\
\hline En rupture d'union & 1,23 & $(0,85-1,77)$ & 1,52 & $(0,96-2,40)$ & $\mathrm{I}, 0 \mathrm{I}$ & $(0,35-2,95)$ & 0,82 & $(0,26-2,6 I)$ \\
\hline
\end{tabular}

Source : Enquête Démographique et de Santé du Bénin, 20I I-20I2

Note : Niveau de significativité : *** $: p<0,01 ;{ }^{* *}: p<0,05 ;{ }^{*}: p<0,10$; Réf. : Modalité de référence ; RRR : Rapport-Risque-Ratio ; $95 \%$ IC : Intervalle de confiance à $95 \%$ 